\title{
APROXIMACIÓN AL ESTUDIO DE LAS CADENAS OPERATIVAS LÍTICAS Y SUS SISTEMAS DE APRENDIZAJE
}

\author{
Approach to the study of lithic operational chains and learning systems
}

\author{
Francisco José Vicente Santos \\ Universidad de Salamanca \\ E-mail: fjvsantos@usal.es
}

Fecha de recepción: 6-III-2011

Fecha de aceptación: 4-IV-2011

\begin{abstract}
RESUMEN: El presente estudio intenta explicar y caracterizar distintas cadenas operativas y gestos técnicos en materiales líticos, así como el método de transmisión del conocimiento y el concepto de aprendizaje. Estos datos nos facilitarían, a posteriori, identificar sobre el material arqueológico la posible existencia de dos tipos de «artesanos». Los dos tipos de artesanos referidos anteriormente son por un lado individuos experimentados en la elaboración de cadenas operativas; y por otro, individuos en etapas de aprendizaje o inexpertos. Los estudios llevados a cabo por distintos investigadores como Karlin, Geneste y otros autores han proporcionado la base para el estudio de este tipo de problemáticas sobre la formación aplicando diversas técnicas para poder reconocerlos.
\end{abstract}

Palabras clave: Aprendizaje, experimentación, talladores inexpertos, talladores expertos.

ABSTRACT: This study attempts to explain and characterize different operating chains, technical gestures in stone, the method of transmission of knowledge and the concept of learning. This experimentation would put differentation bases between "novice"carvers and "experts" carvers.

Keywords: learning, experimentation, carvers operating chains.

\section{INTRODUCCIÓN}

Las investigaciones en materia de industria lítica han sufrido un largo proceso de innovación intelectual y de transformación en sus análisis. La industria lítica, y para ser más exactos los objetos retocados, fueron empleados para diferenciar a los humanos de los animales, aunque este hecho ha sido 
finalmente desechado por las recientes constataciones realizadas en los comportamientos de distintas especies como chimpancés, bonobos y otras especies animales. Estos animales son capaces de valerse de instrumentos para la obtención de comida, utilizar artefactos para "pescar hormigas" (ant fishing) e incluso elaborar "lanzas" rudimentarias para cazar (Goodall, 1964; Serrallonga, 1994, 2005; Pruezt, et alli, 2007; etc ${ }^{1}$ ) estas características son consideradas habilidades socialmente aprendidas y explotadas para una adquisición de recursos que de otra forma sería improbable conseguir.

Los denominados útiles o artefactos se han conceptualizado para el Paleolítico Superior y, como tónica general, para el Paleolítico, como aquellos objetos que han sufrido un retoque. Este hecho, en un principio, no supuso una controversia, pero según han ido avanzando los conocimientos, las investigaciones, y los estudios del Paleolítico, se ha generado una importante polémica entre los investigadores del paleolítico, etólogos y etnólogos. Actualmente, considerar exclusivamente 'útiles' a los objetos retocados supone excluir instrumentos que, sin estar retocados, puedan presentar marcas de uso, o artefactos que por su contexto pueden señalarnos una conceptualización de su posible utilización. La postura tradicional de considerar exclusivamente 'útiles' aquellos que están retocados, en el presente está obsoleta (Eiroa, 2006), es una actitud poco realista y empírica con los datos que nos están aportando disciplinas como la traceología. Esta disciplina reconoce para algunos casos los usos de los útiles y los gestos y acciones con los que fueron empleados, ya sean simples lascas para cortar carne, u otros productos como los percutores que no necesitan modificación.

Por otro lado, el término útil ha generado poca discusión en su conceptualización y definición. Este hecho se agrava y es más preocupante para la historiografía española, que tan solo tiene unas pocas líneas dedicadas al término en manuales o diccionarios (Serrallonga, 1994; Calvo-Trías, 2007). Estos útiles, que se han conceptualizado únicamente como aquellos objetos retocados de piedra, han sido habitualmente esgrimidos y aún hoy se siguen utilizando como forma de datación relativa. Esta datación se realizaba a través de la denominada datación relativa por fósil director. La datación proviene de las cronologías relativas de fósil director de la Geología. Esta forma de datación que, por otra parte, esta imbricada con la tipología, con la que se sostenía que el morfotipo de un útil determinado pertenecía a una cultura y época determinadas.

El propio Bordes (1969), uno de los más importantes y prestigiosos tipólogos (Serrallonga, 1994), señala que la tipología, donde tiene el origen

1 Para información audiovisual utilícese : <http://www.youtube.com/user/ JaneGoodallInstitute\#p/search/0/inFkERO30> y $<$ http://www.youtube.com/user/ JaneGoodallinstitute> 
gran parte del concepto de útil (Calvo-Trías, 2007), es simplemente un sistema que nos sirve para la comparación de distintas estratigrafías y para la correlación de los análisis palinológicos entre diferentes yacimientos.

Las dataciones relativas con fósil director todavía se realizan como modo de aproximación a las posibles cronologías, y se usan normalmente en ausencia de materia orgánica con la que podamos datar el yacimiento de forma absoluta.

Por lo tanto, y gracias al desarrollo de las dataciones absolutas, la tipología ha perdido en gran medida el valor diagnóstico que tenía anteriormente. La revolución cronológica, que en los años finales de la década de los 70 y los iniciales años de la década de los 80 sacudió las investigaciones con las dataciones absolutas generó novedosas expectativas en las investigaciones del Paleolítico (Serrallonga, 1994; Calvo-Trias, 2007). Las novedosas formas de datación han permitido enfocar y diversificar los esfuerzos hacia otras materias de estudio, generando un interés por disciplinas que habían sido poco desarrolladas, como las experimentaciones tecnología lítica y ósea, traceología, arqueozología, etc.

Esta diversificación en los esfuerzos sobre los estudios de la vida en el Paleolítico, dio como resultado un mayor interés en ahondar en estudios sobre tecnología, paleoambiente, arqueozología, y traceología (Calvo-Trías, 2007). Este conjunto de disciplinas, unido a innovadoras ideas aportadas desde la etnología, han fundamentado y desarrollado términos, conceptos y estudios como las "cadenas operativas líticas" o los componentes psicológicos que son necesarios para la elaboración de las mismas (Martínez et alli, 1998; Calvo-Trías, 2007; Geribás, et alli; 2010).

\section{LAS CADENAS OPERATIVAS SU CONCEPTO Y SU REALIDAD SOCIAL.}

Las cadenas operativas son un tipo de conceptualización de las investigaciones que asocia los análisis tecnológicos y traceológicos, aunándolos con el fin último de explicar todas las fases de elaboración de un útil o el tipo de talla de un determinado yacimiento y época. El fin es la explicación global y general de los procesos que intervienen en la misma: desde la adquisición de materia prima, la elaboración, su utilización, hasta el desechado, procesos que la mayoría de los artefactos o de "soportes" de talla (lascas, hojas, etc.) sufren a lo largo de su vida ${ }^{2}$.

Este término/concepto de cadena operativa inicialmente fue desarrollado en la etnología, y fue acuñado por primera vez, por A. Leroi-Gourhan (1970) (Chaînes opératoires) apoyándose en los estudios anteriores de Mauss (Cortés-Sánchez, 2007). Este término/concepto designa actualmente los procesos o secuencias de obtención de materia

2 Nuestro concepto de artefacto/útil es mucho menos restrictivo que el propuesto por la historiografía tradicional, y se asemeja en gran medida a la propuesta de Serrallonga (1994), aunque con ciertas diferencias referidas al contexto arqueológico de estudio. 
prima, elaboración, técnicas ${ }^{3}$, tecnologías ${ }^{4}$, utilización y desecho que tiene un determinado instrumento, pero también la trasmisión del conocimiento de talla y el tratamiento del material lítico.

El concepto ha sufrido cambios desde su origen hasta la actualidad. Podemos reconocer, como hacía Leroi-Gourhan (1964), que es un tratamiento individual y singular de cada tallador. Las técnicas de talla son un procedimiento en el que, generalmente, solo interviene una persona, y sólo es esta persona quien actúa sobre la materia prima, a no ser que sea un núcleo, una lasca o un útil reutilizado en otro momento. No obstante, este procedimiento es también un proceso social en el que intervienen talladores expertos y talladores noveles, trasmitiéndose el conocimiento de unos individuos a otros (Martínez, et alli, 1998:14) siendo en último fin un proceso de sociabilización del grupo. Las investigaciones y estudios que realizamos de las sociedades paleolíticas no pueden ser explicadas, entendidas ni comprendidas como elementos estáticos, ni en sus sistemas y mucho menos por los individuos que las forman (Piegot, 1991).

La evolución del aprendizaje se desarrolla bajo las "enseñanzas" de, por lo menos, un individuo, siendo éste un tallador experto; y otro u otros que reciben la "información", es decir tallador o talladores noveles o inexpertos. Podemos distinguir, por un lado, a un emisor, y por otro a un receptor o receptores. Este contexto y las formas de savoir-faire son el origen y el producto de una tradición socialmente aprendida en lo referente la talla y las cadenas operativas (Martínez et alli, 1998:14). La especulación entorno a la trasmisión de estos conocimientos es bastante controvertida, pero como señala Karlin (1991) el traspaso de conocimientos entre unos individuos y otros se realiza a través de la imitación y la práctica empírica. Estos procesos en la actividad también se han observado en sociedades cazadorasrecolectoras estudiadas actualmente. Completando a lo anteriormente explicado, podemos añadir que, según algunos investigadores (Geribás, et alli; 2010), y por nuestras propias experimentaciones, la talla no es algo innato, previsible o factible de realizar sin un aprendizaje previo o sin unas nociones básicas de los acciones a seguir. La inversión de tiempo, esfuerzo y materia prima, que es necesaria para personas que empiezan a tallar, sin ayuda de un tallador "experto", es muy superior a la necesaria con la ayuda de un iniciado a la talla.

Este aprendizaje, en especial en la elaboración de artefactos que requieren una talla tan especializada como los foliáceos, necesita un largo proceso de amaestramiento. La reducción bifacial quizá sea uno de los procesos más complejos dentro de la talla, que se puede dividir en tres fases (Maíllo, 1999:205):

3 Acepción 5 de la RAE: "Conjunto de procedimientos y recursos de que se sirve una ciencia o un arte".

4 Acepción 4 de la RAE: "Conjunto de los instrumentos y procedimientos [...] de un determinado sector o producto." 
Tomando como base esta teoría entendemos que:

1. "SCHEMA: representación mental de la producción lítica, es decir, los pasos o fases teóricas de los diferentes métodos de talla. Es algo cultural y común para toda la comunidad".

El Schema es la idea de los pasos tecnológicos o etapas de la cadena operativa necesarias para la elaboración de un determinado artefacto. Esto significa que el artesano conoce la teoría (Pelegrin, et alli, 1988; Karlin, 1991). Este es el proceso previo para poder realizar el artefacto o la talla deseada.

2. "SCHEME: proyecto del tallador de esa representación mental cuando tiene el nódulo de materia prima en la mano".

El Scheme hace alusión a la teoría y proyectarla sobre un nódulo o sobre un soporte de talla que pueda facilitar la fabricación del artefacto deseado (Pelegrin, et alli, 1988; Karlin, 1991). Este proceso es el momento en el que por un lado se necesita la teoría, pero también la aplicación práctica, en tanto en cuanto el conocimiento de un tallador experto puede hacer que se reconozcan mejor las posibilidades de un nódulo, núcleo o soporte para la elaboración de determinados proyectos. Siempre teniendo en cuenta que el soporte o el nódulo y la materia prima no tienen por qué determinar el producto final (en especial si el tallador es experto y conoce la materia prima).

3. "SEQUENCE: trabajo del nódulo que pueden describirse dentro de las fases de la cadena operativa".

Y la Sequence, la última fase, es el trabajo práctico de un nódulo o un soporte es el elemento con el que podemos diferenciar a los talladores noveles de los expertos, no solo porque quizá es donde se halle la mayor diferencia, sino que también son elementos constatables. Esta fase es un proceso más complejo de lo que nos pensamos, ya que, aunque conozcamos perfectamente los dos pasos anteriores, estos no sirven de nada a la hora de intentar aplicarlos por primera vez (Sequence).

Estas son las tres fases del conocimiento y realización necesarias para la elaboración de un artefacto, con posibilidad de que pueda ser utilizado.

En la actualidad, estas fases se han reducido en gran medida en nuestros sistemas de enseñanza, siendo la primera la única que conocemos, es decir, la teoría. Las otras dos fases se han difuminado en mayor o menor medida en la primera, hasta el inicio de la vida laboral. Piegot (1991) afirma, con respecto al aprendizaje, que no debemos caer en las simplificaciones universalistas sobre la enseñanza. El aprendizaje como tal, quizás sea necesario reconocerlo, ha perdido la significación que poseía en las sociedades paleolíticas. Algunos investigadores defienden que la cognición humana se desarrolla paralelamente a la utilización y la creación de herramientas (Foley y Lahr, 2003). Estas afirmaciones deberían ser verificadas actualmente con las investigaciones realizadas a primates en estado salvaje (Serrallonga, 1994, 
2005; Pruezt, et alli, 2007) que nos proporcionarían cierta fiabilidad de estos procesos y de la utilización y la elaboración de herramientas. No obstante, aún pudiendo acercarnos a esta problemática, con las investigaciones realizadas en etología, debemos recordar que la equivalencia entre australopitecos y primates actuales no es una comparación al 100\% certera, y esto se agrava si lo trasladamos a nuestra propia especie (Piegot, 1991) por la complejidad cultural y la gran diversidad de esta.

Distintos investigadores (Karlin, 1991; Harris, 2008; etc.) señalan en sus estudios etnológicos cómo los grupos de cazadores-recolectores realizan su aprendizaje con comunicación no verbal, es decir, a través de la enseñanza visual, y especialmente mediante la imitación. Sabemos que, quizá, las sociedades actuales y las paleolíticas no son exactamente iguales, pero los datos etnológicos son una información a tener en cuenta (Piegot, 1991). Nuestro objeto de investigación es, precisamente, el aprendizaje de estas sociedades.

\section{LA EXPERIMENTACIÓN}

\subsection{Muestra Para la eXPERIMENTACión}

La aproximación experimental al aprendizaje básico de talla se ha realizado con un total de dieciocho individuos, con ninguna experiencia o con escasas nociones sobre la talla lítica. Aunque, esta muestra se ha tomado de entre los alumnos de la licenciatura/grado de Historia de la universidad de Salamanca, debido a que tienen unas nociones básicas de Prehistoria, y más concretamente sobre el Paleolítico.

Podemos dividir el total de los individuos en dos grupos en función de sus conocimientos relativos sobre la materia, tanto en cuanto 14 de ellos, es decir, tres cuartos del total pertenecen al $1^{\circ}$ curso del grado de Historia, mientras que el resto, un cuarto de la población total que configura este experimento pertenecen a los últimos cursos de la Licenciatura de Historia.

Los datos concretos sobre la edad, si bien en ciertos proyectos resulta relevante para el resultado final, en esta ocasión parecía un dato menos relevante que saber sus conocimientos previos.

La distribución de participantes por sexos es el siguiente: seis mujeres y doce hombres, suponiendo un tercio y dos tercios, respectivamente, de cada sexo.

Diez de los talladores no habían tenido ninguna práctica con la talla, y los otros ocho tan sólo habían practicado única una vez con sílex.

Todos los individuos sí habían visto a personas tallar como algún video de talla. Este dato nos parecía muy importante, ya que puede facilitar la compresión o la modificación de patrones de movimientos y tratamiento del nódulo. 


\subsection{Metodología de LA EXPERIMENTACión}

Estos talladores eran todos noveles, y se realizó de forma individualizada la experimentación. Esta práctica no se grabó por dos razones: la primera de ellas, por la falta de consentimiento de todas las personas, que realizaban la prueba y por las características simples de la misma que hacían poco necesaria la segunda visualización de la experimentación. Sí que realizamos fotografías de la mayoría de los participantes en distintos momentos de la experimentación ${ }^{5}$.

La prueba de talla constaba de dos etapas básicas: una de fondo teórico, en la que buscábamos saber los conocimientos sobre el Paleolítico que tenía cada uno de los individuos, y por otro lado la segunda etapa respondía a la parte práctica, en la evaluábamos la capacidad de cada persona para realizar dos tipos de retoque (escamoso y abrupto) y la extracción de soportes como una lasca o de un nódulo de sílex informe.

Las preguntas de teoría eran las siguientes:

1. ¿Qué es un retoque?

2. ¿Definición de retoque escamoso y abrupto?

3. ¿Qué tipos de percutores hay?

4. ¿Qué es una lasca y qué es una lamina/hoja?

La primera pregunta, sobre la definición del retoque: el 100\% de los participantes supieron explicar y enumerar distintos tipos de retoque.

La segunda pregunta sobre la variedad de retoques: la totalidad de los casos, los estudiantes no sabían cómo eran físicamente los retoques preguntados, pero sí sabían reconocerlos en una diapositiva o dar una aproximada definición de los mismos.

La tercera pregunta en torno a los percutores: el 100\% sabía y reconocía percutores blandos y duros.

La cuarta pregunta: el 100\% expresaba correctamente una definición de lasca, pero tan solo él $80 \%$ de los estudiantes sabía diferenciar correctamente una hoja de una lasca.

La experimentación se realizó con los talladores sentados y dándole a elegir entre doce percutores de distintas materias y peso. Los doce percutores y los dos presionadores se dividían de la siguiente forma: cuatro percutores duros, de cuarzo o cuarcita (uno de tamaño pequeño, dos medianos y uno de gran tamaño), seis percutores semi-blandos de arenisca o caliza (tres de pequeño tamaño, dos de tamaño medio y uno de gran tamaño) y se les proporcionó también dos percutores blandos (uno de gran tamaño y otro de pequeño

5 Queríamos agradecer la inestimable e incondicional ayuda aportada por Paula Ortega en la realización de las fotos. 
tamaño) y por último, dos presionadores (Fig. 1). Posteriormente se le proporcionó una lasca anteriormente extraída por otro tallador. Se les invitó a que realizasen un retoque abrupto y un retoque escamoso sobre distintas partes de la misma lasca.

Los talladores, tanto para el retoque abrupto como para el retoque escamoso, optaron por utilizar percutor semi-blando, para el retoque abrupto once y para el retoque escamoso diez, en ambos casos el porcentaje es superior al 50\%.

El retoque abrupto fue elaborado o al menos aproximado en su elaboración con presionadores por dos de los participantes y uno optó por un percutor de cuarzo. En cuanto al retoque escamoso en cambio sólo tres participantes lo intentaron con presionador y nuevamente uno sólo de los individuos lo intento con percutor duro.

La mayoría de los participantes no consiguieron realizar un retoque pleno en ninguno de los dos casos, aunque sí es cierto que el retoque abrupto fue aproximado por varios talladores, pero sin un pleno desarrollo del mismo. Esto nos plantea cuestiones sobre la facilidad de hacer este tipo de retoque.

Con esta experimentación quedó claramente diferenciado, por una parte, que la teoría y los conocimientos en Paleolítico son temas no necesariamente relacionados con la realidad del retoque, y por otra se reconoció que el retoque no es una cuestión de intuición.

El siguiente de los apartados es la talla lítica o extracción de lascas y hojas. Esta fase de la experimentación se elaboró a través de cuatro etapas. La primera etapa fue la elección de un núcleo sin una forma predeterminada, es decir, informe y un percutor. Es interesante explicar qué tipos de percutor eligieron, cómo los agarraban y cómo percutían enlazando con la fase siguiente de la experimentación.

La segunda de las fases era obtener, sin ningún tipo de ayuda y con las nociones que tuvieran, una lasca o una hoja.

La tercera de las etapas constituía a nivel explicativo y verbalizado qué errores estaban cometiendo y cómo debían subsanarlos sin ningún gesto, tan solo de forma oral.

Por último, se les ayudaba con comunicación verbal y no verbal para reconocer hasta qué punto se podían imitar las tácticas y técnicas de explotación del individuo experimentado.

\section{Resultados}

Sobre la elección de los percutores y núcleos en la parte inicial de la práctica, es importante tener en cuenta que a todos los individuos se les daba total libertad para elegir cualquiera de los percutores y de los núcleos. La mayoría de ellos elegían el núcleo sin pararse a mirarlo y sopesar la calidad. 
No se paraban a pensar, ni a comparar con otros nódulos; simplemente escogían el más grande, el más cercano, etc. Este proceso nos pareció destacable, ya que todos los individuos han visto tallar en vivo y a través de medios audiovisuales y en ningún momento se pararon a pensar cuál podría tener menos impurezas, mejor textura, mejores ángulos.

La mayoría de los participantes comenzaron cogiendo el núcleo antes que el percutor, a excepción de dos participantes que escogieron el percutor antes que el nódulo. Podemos destacar que ninguno de los individuos eligió el percutor blando, siendo éste, por lo general, un percutor de mejores características para la talla. Por último, salvo tres individuos, el resto escogieron el percutor de una forma "aprisionada" y rígida, más parecida a la manera de agarrar una piedra para lanzarla que para tallar con ella (Fig. 2).

La segunda etapa ya ha sido parcialmente revelada anteriormente, y es la utilización de percutores sin ningún tipo de criterio. Algunos casos era más que evidente la desproporción entre el percutor y el nódulo trabajado. Estos procesos son un ejemplo de la necesidad de unos conocimientos básicos sobre las proporciones del nódulo y el percutor. Este hecho es muy importante para evitar algunos tipos de errores técnicos en el nódulo (Baena, 1998).

Por otro lado, también es reseñable que uno de los participantes intentó aplicar el retoque en la lasca a través del golpeo sobre uno de los percutores de arenisca. Los errores de ejecución eran muy diversos, y tan sólo con cuatro o cinco golpeos se podía comprobar que muchos de ellos tenían errores, como la mala colocación del núcleo y posición en la mano o en la pierna, la errónea manipulación del percutor y del propio núcleo, los errores graduación del ángulo de percusión, etc. Todos estos errores provocaron que tan solo uno de los dieciocho individuos pudiera obtener una lasca, y otros cuatro más obtuvieran elementos de características bastante amorfas (Fig.3). Los problemas se podían observar en el propio núcleo, donde se advertían astillamientos de las zonas de talla, casos de reflejados, etc.

Esta primera fase puede destacar especialmente por la escasa cantidad de personas que obtuvieron un producto adecuado (tan solo una persona) y por la gran cantidad de defectos, tanto técnicos como de talla, observados en los participantes. Esto generaría no solo problemas a la hora de obtención de productos, sino también en el aprovechamiento de los núcleos.

La siguiente de las etapas es una introducción básica y explicativa de los datos técnicos necesarios para la obtención de lascas y láminas. Las explicaciones orales se realizaron mediante un discurso "normalizado" para todos los participantes, pero se añadían ciertas explicaciones específicas de errores destacados que se observaban en cierto tallador.

Este discurso implicaba temas como la sujeción del percutor, el tamaño de éste con respecto al nódulo, el ángulo de trabajo (siempre tiene que ser menor a $90^{\circ}$ ), localización y posturas para la talla. Todos, o al menos 
la mayoría de los individuos, tenían defectos generalizados como la forma de empuñar el percutor, la colocación del núcleo, la forma de percutir, a veces por golpeos en exceso en el interior de la pieza y por lo tanto sin obtención de lascas, a veces por exceso en el golpeo en la zona exterior provocando astillamientos, etc.

La última fase experimental se realizaba con los participantes y ayudándoles en la elaboración de un soporte. Las explicaciones verbalizadas se acompañaban con otras prácticas como colocación del núcleo, demostración gestual de movimientos, etc. Todo esto consiguió que los participantes obtuvieran un soporte de lascado.

\section{LOS DATOS Y APORTACIONES OBTENIDOS}

Las conclusiones y datos extraídos de la experimentación han sido variados y tan sólo aproximados a la realidad por lo que hay que tomarlos con cautela. Se han podido reconocer ciertos patrones de aprendizaje reflejados y astillamientos en los núcleos, aunque esto sólo es una realidad parcial debido a la necesidad de experimentar los mismos hechos sobre otros tipos de materias primas y calidades. Esta variabilidad de calidades en la materia prima puede provocar una alteración del registro. Un tallador, aún siendo experto, puede generar gran cantidad de defectos de talla, si el sílex que trabaja es poco conocido y de mala calidad.

Los patrones y defectos de talla de principiantes son astillamientos del borde del núcleo, reflejados, roturas en cuña, que generalmente no se presentan en un tallador experto y con un sílex de buena calidad.

La experimentación nos ha servido para descartar la facilidad de tallar soportes sin un aprendizaje previo; también se han comprendido mejor los procesos de amaestramiento y la inutilidad de verbalizar un discurso para la enseñanza de la talla. La facilidad en la enseñanza práctica, y en la compresión de la misma hacen del sistema práctico la mejor forma de aprendizaje. Esta experimentación nos ha llevado a comprender el hecho de que en muchos pueblos de cazadores-recolectores (Karlin, 1991) los jóvenes imiten a los adultos en un intento de aprender una labor determinada, sin la necesidad de expliquen de forma verbalizada los procesos de aprendizaje.

Los procesos y el aprendizaje de la talla, así como la obtención de soportes, es un proceso "fácilmente" asimilable y sencillamente imitativo. Esto genera una dificultad a la hora de poder diferenciar los dos tipos de talladores y su experiencia. En objetos que requieren mayor pericia técnica como las hojas de laurel es mucho más sencillo. Las mejores características para la diferenciación entre talladores es el núcleo desechado, ya que en ellos se han desarrollado tanto problemas de astillamientos, como reflejados y sobrepasados. Todos estos datos nos pueden ayudar a diferenciar a la experiencia de cada uno de los talladores. 


\section{Bibliografía:}

Bordes, F. (1969): "Reflections on typology and technology in Paleolithic" Artic Anthropology 6: 1-29.

Cortés-Sánchez, M. (2007): El paleolitico medio y superior en el sector central de Andalucía (Córdoba y Málaga) Madrid : Ministerio de Cultura, Subdirección General de Publicaciones, Información y Documentación, D.L.

Eiroa, J. J. (2006): Nociones de prehistoria general. Barcelona, Ariel.

Foley, R. y Lahr, M. M., (2003): “On stony ground: lithic technology, human evolution, and the emergence of culture." Evolutionary Anthropology 12, 109-122.

Geribàs, N.; Mosquera, M. y Vergès J. M. (2010): "What novice knappers have to learn to become expert stone toolmakers" Journal of Archaeological Science, 37: 2857- 2870.

Goodall, J. (1964): "Tool-using and aimed throwing in a community of free-living cimpanzees", Nature, vol. 201, no 4.926:. 1264: 1266.

Harris, M. (2008): Nuestra especie. Salamanca, Alianza editorial. Primera edición 1989.

Karlin, C. (1991): "Connoisances et savoir-faire: comment analyser un processus technique en prehistoire. Introduction.” En; Mora, R.; Terradas, X.; Parpall, A. \& Plana, C. (Eds.): Tecnología y cadenas operativas líticas. Treballs de arqueología, 1: 99-1.

Leroi-Gourhan, A. (1970): Le geste et la parole. Vol. I, Technique et langage, Paris: Albin Michel.

Maíllo-Fernández, J. M. (1999): "Esquemas operativos y conocimiento técnico: el caso del yacimiento solutrense del valle Almoinha (Torres Vedras, Portugal)." Espacio tiempo y forma, 12: 185-214.

Martínez-Fernández, G. y Afonso, J. A. (1998): “La producción lítica: un modelo para el análisis histórico de los conjuntos arqueológicos de piedra tallada". En: Bernabeu, J.; Orozco, T. y Terradas, X.: Los recursos abióticos en la prebistoria (caracterización, aprovisionamiento e intercambio) Ed. Universidad de Valencia.

Pelegrin, J.; Karlin, C. y Bodu, P. (1988): Chaînes opératoires: un outil pour le préhistorien. Technologie Préhistorique. Notes et Monographities techniques, n. $25:$ 55-70.

Pigeot, N. (1991): 'Réfiexions sur prehistoire technique de L'Homme: de i'evolution cognitive a l'evolution culturelle.” Paleo, 3: 167-199.

Pruetz, J. and Bertolani, P. (2007) "Savanna Chimpanzees, Pan troglodytes verus, Hunt with Tools” Current Biology 17, March 6: 412-417.

Serrallonga i Atset, J, (1994) "Homo Faber, el fin de un mito: etología 
y Prehistoria, una aproximación al presente para reconstruir el pasado del "útil"'" Pyrenae: revista de prehistòria $i$ antiguitat de la Mediterrània Occidental, No. 25: 31-49.

- (2005): "No estamos solos: austrolopitecos y chimpancés habilidosos.". Existo, Luego Pienso. Los Primates y La Evolución de la inteligencia bumana. En Guillén-Salazar, F. (Ed.) Ateles Editores

Agradecimientos:

Queríamos agradecer a todos los participantes de esta experimentación su labor, sin la cual no habría sido posible realizar esta aproximación, y a María Soledad Corchón Rodríguez por sus aportaciones. 


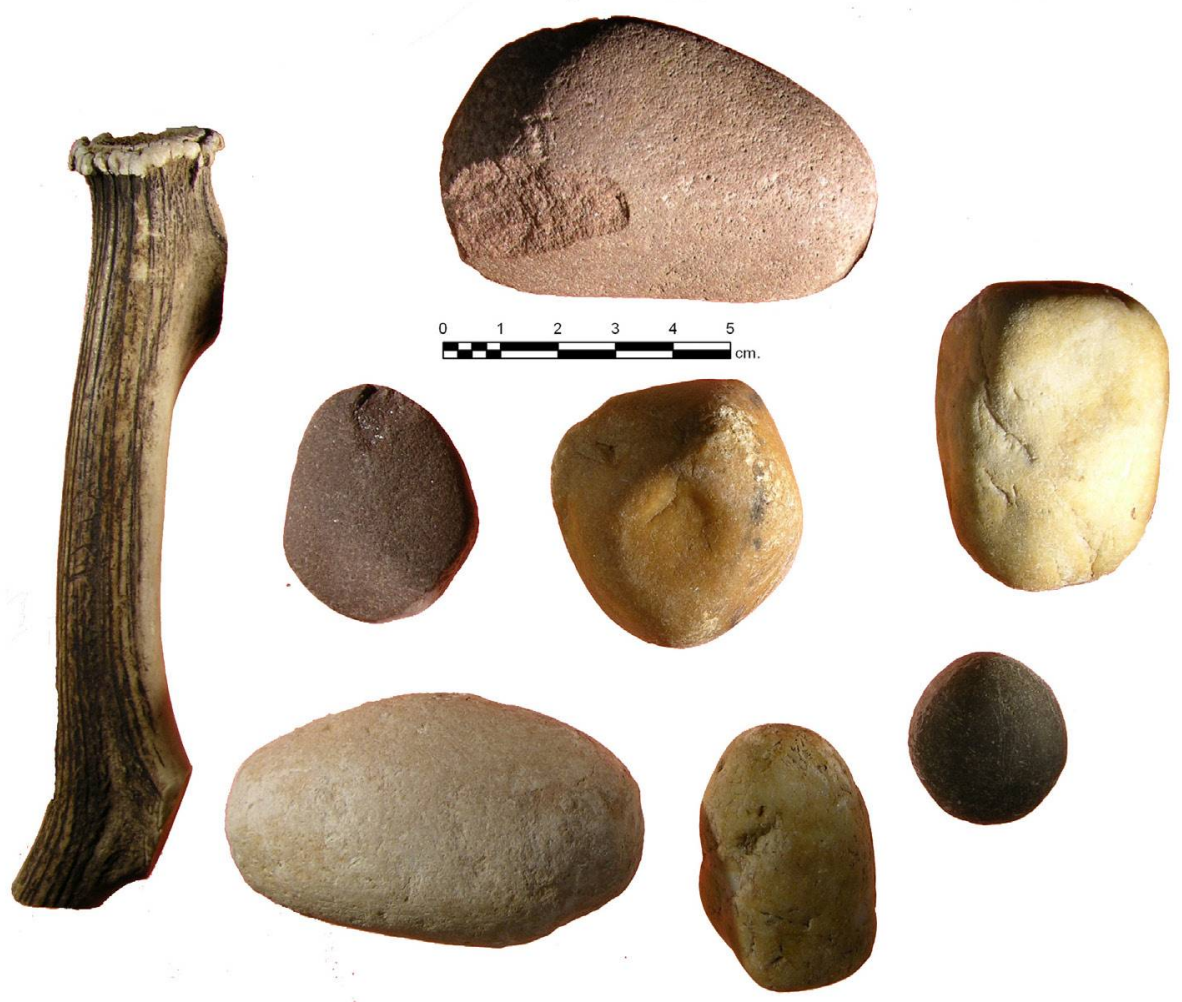

(Fig. 1). Algunos de las opciones de percutores que los participantes podían elegir.

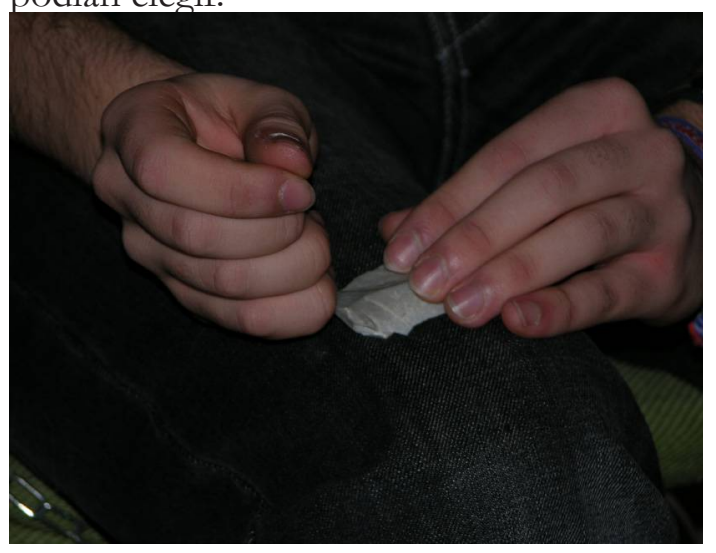

(Fig. 2). Tallador inexperto que coge el percutor de forma aprisionada y "rígida" e intenta realizar el retoque sobre la pierna. 


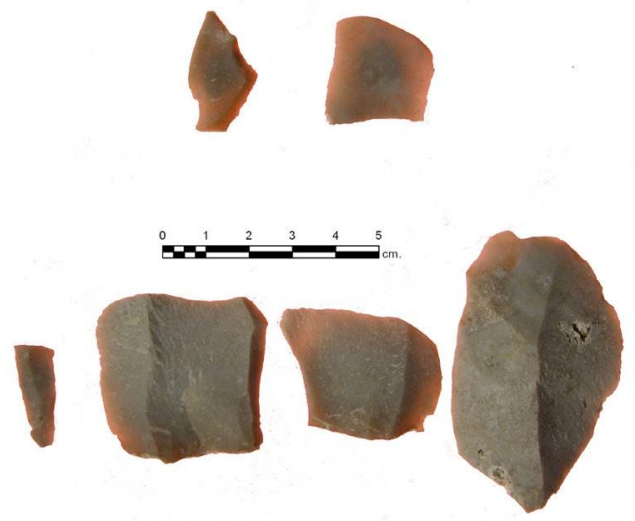

(Fig.3). Productos informes de talladores en la primera fase de la experimentacióny productos obtenidos en laúltima fase dela experimentación. Parte superior y parte inferior de la fotografía respectivamente.

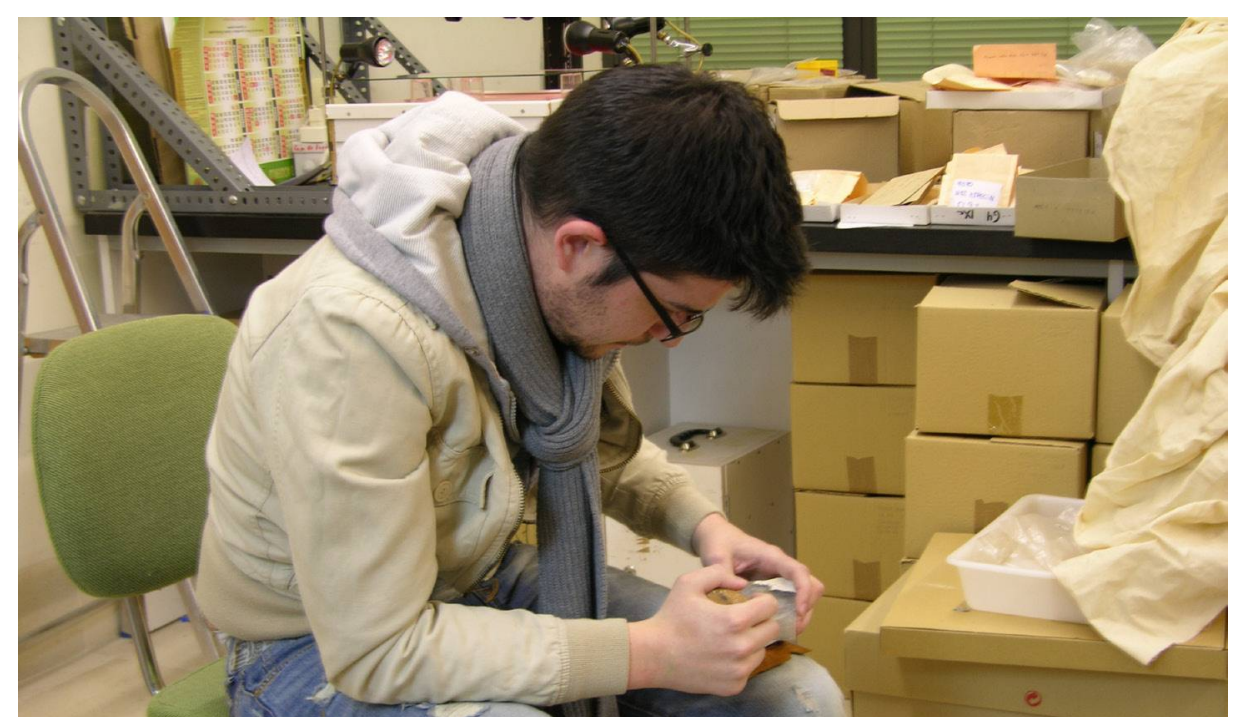

(Fig.4) Tallador inexperto con defectos visibles en su forma de agarrar el percutor y colocar el núcleo. 


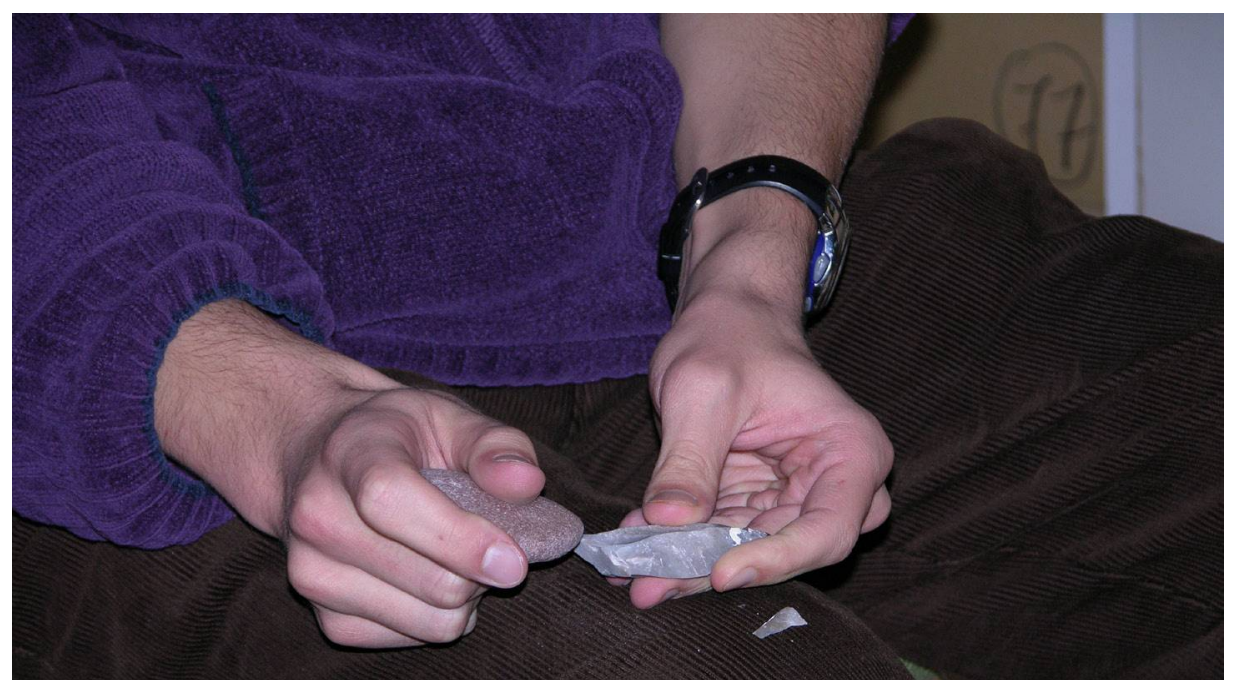

(Fig.5) Error en la colocación de lasca para realizar el retoque.

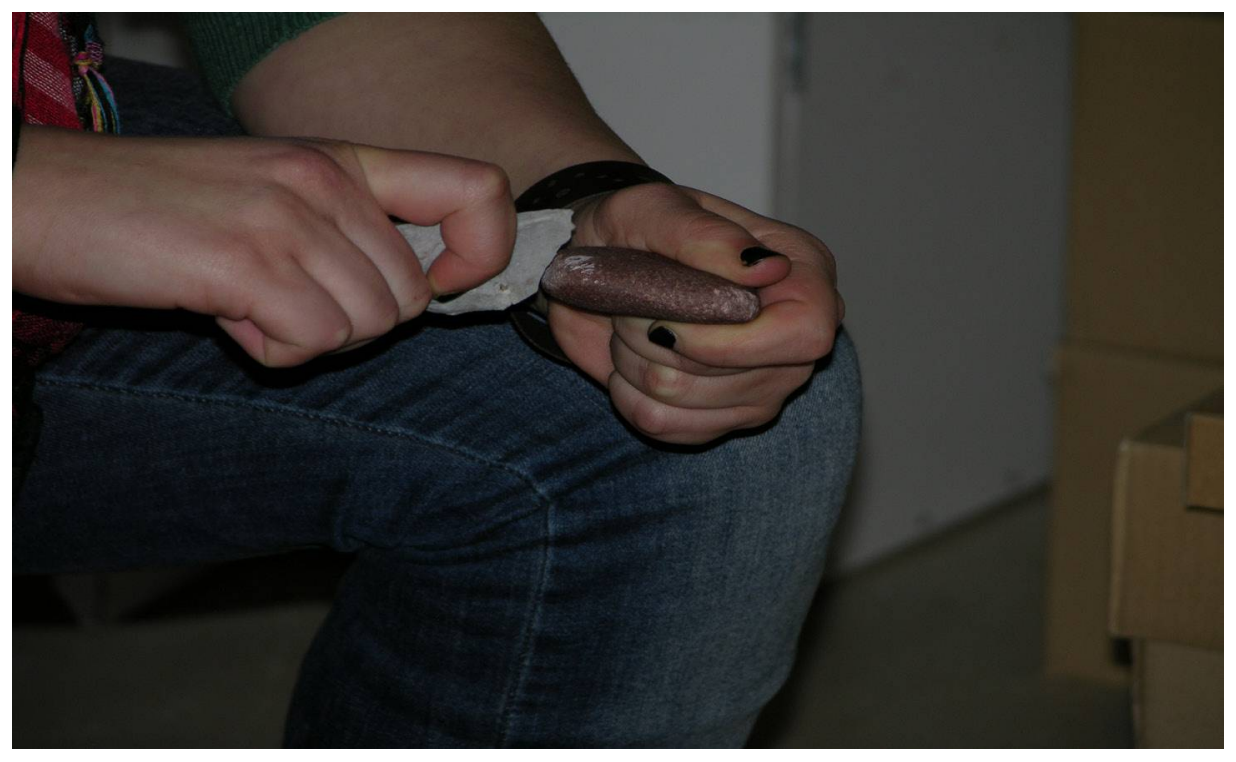

(Fig. 6) Intento de realización de retoque golpeando la lasca contra el percutor. 


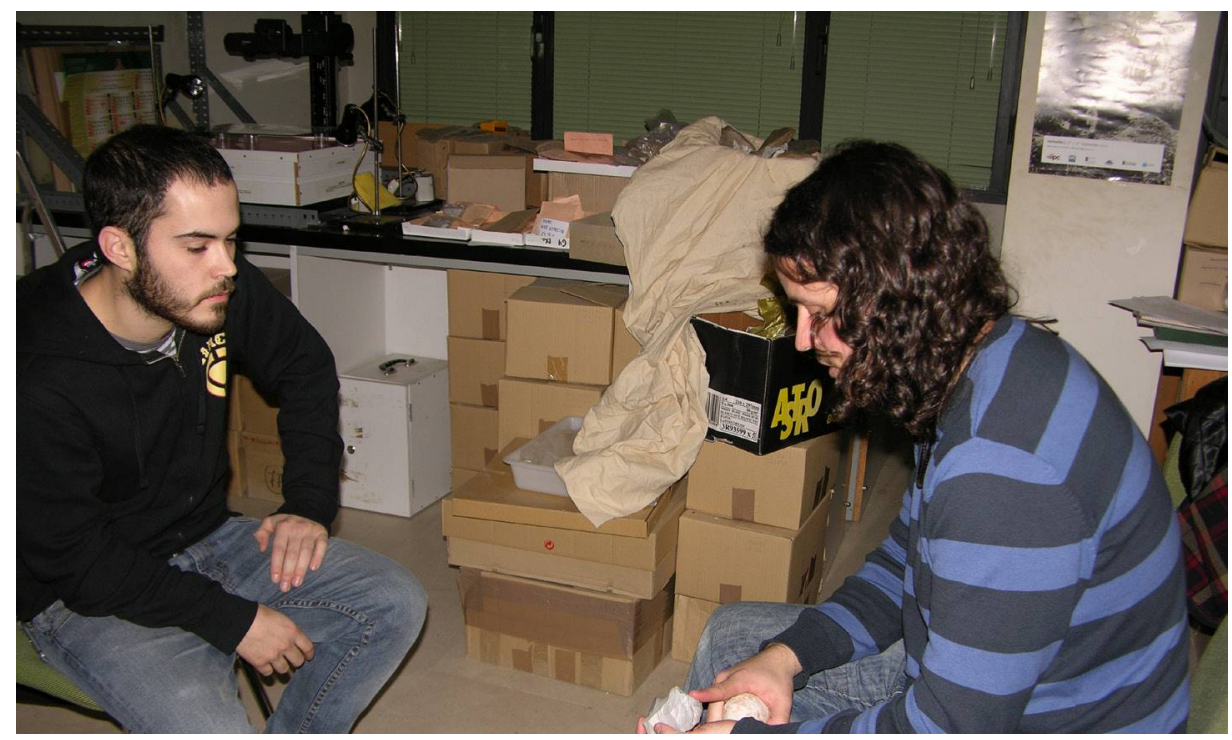

(Fig.7) Última fase de enseñanza donde se les impartían lecciones verbalizadas y prácticas.

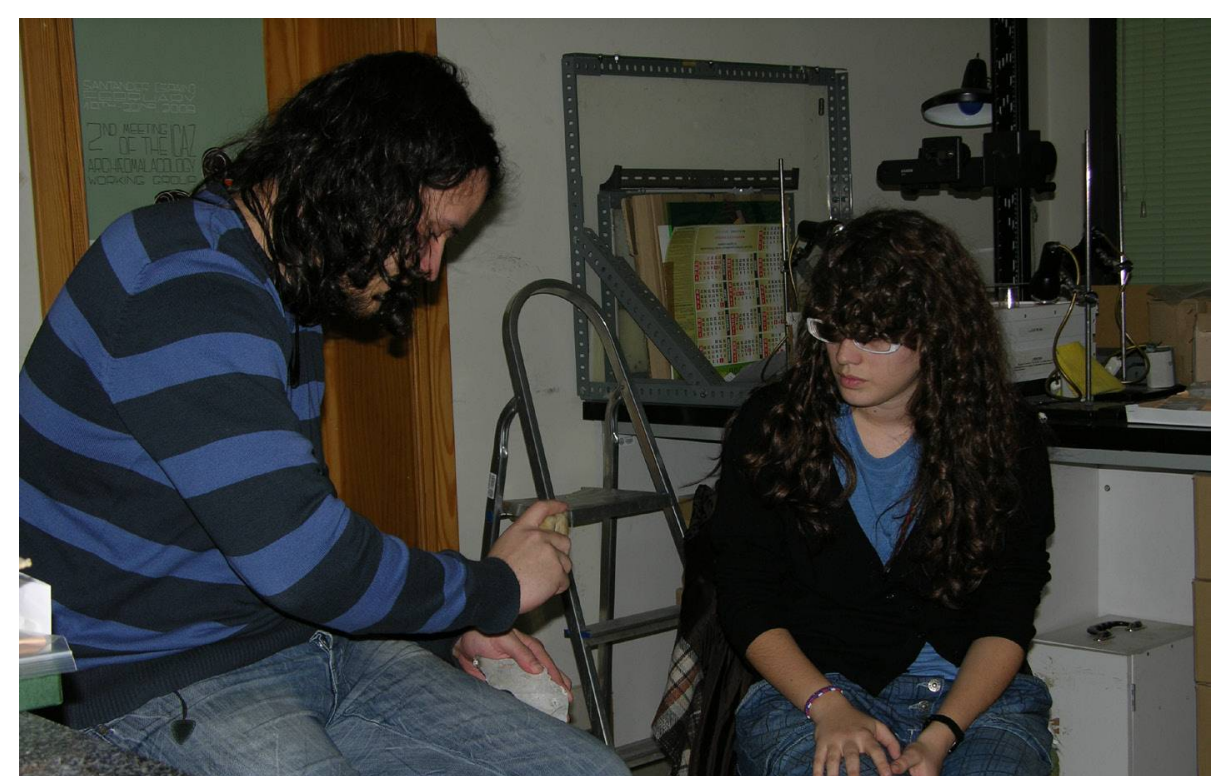

(Fig. 8) Explicación Teórico-práctica sobre errores cometidos en las anteriores fases de la experimentación. 